\title{
An Electromagnetic Circuit Simulator For Power Electronics
}

\author{
Jeroen Zwysen, Pieter Jacqmaer, Ratmir Gelagaev, Johan Driesen \\ ESAT-ELECTA, K.U.Leuven; \\ Kasteelpark Arenberg 10, bus 2445, 3001 Leuven-Heverlee, Belgium \\ Jeroen.zwysen@esat.kuleuven.be, pieter.jacqmaer@esat.kuleuven.be, ratmir.gelagaev@esat.kuleuven.be \\ johan.driesen@esat.kuleuven.be
}

\begin{abstract}
A method for solving the full equations of Maxwell for circuits with discrete non-linear, non-ideal elements is presented. To this end the method of moments is used in combination with a classical circuit simulator. Both operate in the frequency domain. A few methods to greatly accelerate the calculations are also presented. The methods are implemented and tested in Matlab ${ }^{\circledR}$.
\end{abstract}

\section{INTRODUCTION}

With the upcoming of wide bandgap semiconductors, transistors in power electronics can be used with higher switching frequencies, up to several tens of megahertzes. In the rising and falling edges of the pulse generated by transistors even higher frequencies are present. At these frequencies the conductors in the circuits will act as transmission lines. This means reflections can occur in case of poor impedance matching which can double the voltages across the electrical components. Also energy will be lost due to radiation. This radiation can interact with neighbouring circuits. This means that classical circuit solvers like SPICE cannot simulate these circuits accurately at high switching frequencies.

Nowadays commercial software exists that can solve the full equations of Maxwell for power electronics. They mostly use the partial element equivalent circuit method (PEEC) [1]. Examples are CST Studio and Cedrat's InCa3D. Often times the PEEC method is used in combination with a classical circuit simulator in the time domain like SPICE. This is good for transient solutions but for electromagnetic interference (EMI) problems mostly a steady-state solutions of the system is desired. This would require the simulation of multiple periods in the time domain. In the frequency domain, a classical circuit simulator will immediately return the steady-state solution. Another big advantage is the ease of incorporation of the frequency-dependent equivalent admittance.

A method for solving the full equations of Maxwell, in the frequency domain, for circuits with discrete non-linear, non-ideal elements, is presented in this paper. The structure of the method is shown in Fig. 1. A buck convertor is used as an example. The conductors of the circuit are modeled in 3D and are connected with discrete circuit elements. The points to which the elements connect will be called nodes. At the start of the calculations the program discretizes the conductors. Afterwards the method of moments (MoM) is applied to find an equivalent, frequency dependent, admittance matrix of the conductors. When this admittance is connected with the discrete circuit elements, the resulting equivalent circuit can be solved with a classical circuit solver. The final results are the voltages at the nodes.

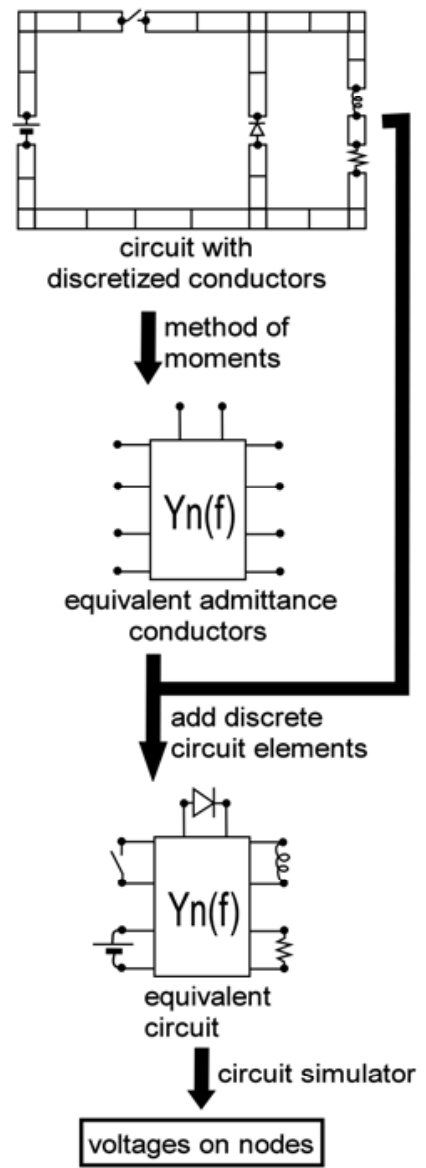

Fig. 1. Structure of the presented method

\section{METHOD OF MOMENTS}

\section{A. Full impedance matrix}

The MoM is widely studied [2][3][4] and only the result shall be given here. After discretizing the model into current and charge elements, applying the MoM will result in the full impedance matrix $\mathrm{Z}$ :

$$
\left[V_{e x t}\right]_{N_{c u} x 1}=[Z]_{N_{c u} x N_{c u}}[I]_{N_{c u} x 1}
$$

In equation (1) $\mathrm{N}_{\mathrm{cu}}$ is the number of current elements, $\mathrm{V}_{\text {ext }}$ is a vector with the external voltages applied across every current element and $I$ is the current through every current element. The full impedance matrix $\mathrm{Z}$ is frequencydependent. Its frequency-dependence originates from the following Green functions that will be calculated $\mathrm{N}_{\mathrm{cu}}{ }^{2}$ times:

$$
G(\vec{p}, V)=\frac{1}{4 \pi V} \int_{V} \frac{e^{-j k r}}{r} d V .
$$


In equation (2) $\mathrm{k}$ is the wavenumber, $\mathrm{r}$ is the distance from the given point $\mathrm{p}$, where the fields need to be evaluated, to the integration point inside the volume $\mathrm{V}$. This integration is done numerically and thus consumes a lot of time. As the integrand is a function of frequency the Green functions must be recalculated for every other frequency. But if the dimensions of the current and charge elements are much smaller then the smallest wavelength in the system equation (2) can be approximated as:

$$
G(\vec{p}, V)=\frac{e^{-j k r_{m}}}{4 \pi}\left[\frac{1+j k r_{m}}{V} \int_{V} \frac{1}{r} d V-j k\right] .
$$

In equation (3) $r_{m}$ is the distance from point $p$ to the middle of volume $\mathrm{V}$. Now the integrand of the remaining integral in equation (3) is not a function of frequency anymore. That means the integrals must be evaluated only once and can then be reused for calculating the full impedance matrix at every frequency.

A second advantage of the formulation in equation (3) is that calculating the remaining integral has already been studied and optimized in quasi-static programs like FastCap [5] and FastHenry [6].

\section{B. Node admittance matrix}

Equation (1) gives the relation between the voltages applied over every current element and the current through every current element. But the discrete elements are only adjacent to $\mathrm{N}$ of those current elements, $\mathrm{N}$ being the number of nodes. That means only the relation between voltage across and current through those current elements is required. That relationship is given by the node admittance matrix $Y_{n}$ :

$$
\left[I_{s}\right]_{N \times 1}=\left[Y_{n}\right]_{N \times N}\left[V_{s}\right]_{N \times 1} \text {. }
$$

$I_{s}$ are the currents through the nodes and $V_{s}$ are the voltages at the nodes. The matrix $Y_{n}$ can be found by solving equation (1) for the current vector N-1 times. This consumes time but it greatly accelerates the calculations in the next step, the circuit simulator, as the dimensions of $Y_{n}$ are many times smaller than $\mathrm{Z}$.

\section{Validation}

To validate the first part of the program, the output is compared with an analytical expression for the current in a transmission line. The transmission line consists of two thin, parallel conductors with an AC voltage of one volt applied to one end. Fig. 2 shows the amplitude of the current in the transmission line for three different frequencies. They are compared with the following formulas [4]:

$$
I(x)=\frac{V_{0}\left[e^{-j x \beta}-\Gamma_{L} e^{j \beta(x-2 L)}\right]}{120 \ln \left(\frac{4 D}{w}\right)\left(1+\Gamma_{L} e^{-j 2 L \beta}\right)}
$$

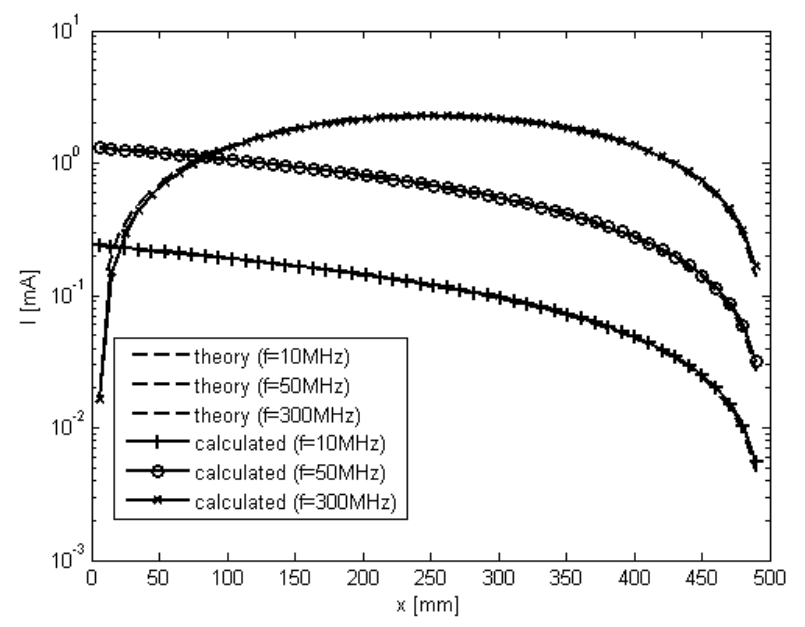

Fig. 2. Amplitude of current in open ended transmission line

A second validation comes from comparing the output of this part of the program with measurements. Measurements of the input admittance of a dipole antenna of length 1, suspended in free space, can be found in [7]. The calculated and measured input admittance of this antenna are shown in Fig. 3.

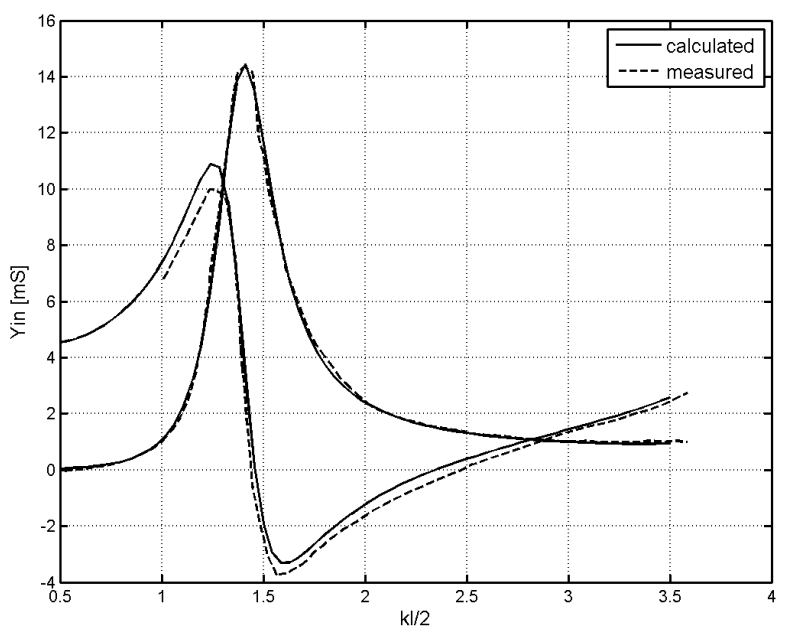

Fig. 3. Input admittance (real ( $\Lambda$-curve) and imaginary part (Z-curve)) of a dipole antenna with length 1

The calculated values match well with the analytical formulas as well as with the measurements of input admittance of the dipole antenna.

\section{Circuit Simulator}

\section{A. Equations}

The circuit simulator in the frequency domain will solve the following non-linear matrix equation [8] iteratively:

$$
[Y]_{2 N H \times 2 N H}[V]_{2 N H \times 1}+\left[I_{s}\right]_{2 N H \times 1}+\left[I_{n l}(V)\right]_{2 N H \times 1}=0
$$

The equations (6) state that the sum of the currents leaving every node must be zero. This must be true for all $\mathrm{H}$ frequencies. Because the equations and unknowns are complex, the real and imaginary part must be separated 
which doubles the size of the matrices. The matrix Y and vector $I_{s}$ contain the contributions of the linear elements to the currents while the vector $I_{n l}$ contains those of the nonlinear elements. To solve matrix equation (6), this nonlinear term will be linearly be approximated at every iteration $\mathrm{k}$ :

$$
\left[I_{n l}(V)\right] \approx\left[I_{n l}\left(V^{k}\right)\right]+\left[J_{n l}\left(V^{k}\right)\right]\left([V]-\left[V^{k}\right]\right) .
$$

From equations (6) and (7) the voltages on the nodes in step $\mathrm{k}+1$ can be solved.

Evaluating the Jacobian $\mathrm{J}_{\mathrm{nl}}$ numerically is very time consuming. The calculation can be accelerated greatly by using the fact that the unknowns are quantities in the frequency domain that represent a time domain signal. Derivation of this method is given in [8] and the outcome is repeated here:

$$
\begin{aligned}
\frac{\partial I_{m, k}}{\partial V_{n, l}} & =\left[\begin{array}{ll}
\frac{\partial I_{m, k}^{R}}{\partial V_{n, l}^{R}} & \frac{\partial I_{m, k}^{R}}{\partial V_{n, l}^{I}} \\
\frac{\partial I_{m, k}^{I}}{\partial V_{n, l}^{R}} & \frac{\partial I_{m, k}^{I}}{\partial V_{n, l}^{I}}
\end{array}\right] \\
& =\left[\begin{array}{ll}
G_{m, n, k-l}^{R}+G_{m, n, k+l}^{R} & G_{m, n, k+l}^{I}-G_{m, n, k-l}^{I} \\
G_{m, n, k-l}^{I}+G_{m, n, k+l}^{I} & G_{m, n, k-l}^{R}-G_{m, n, k+l}^{R}
\end{array}\right]
\end{aligned}
$$

In equation (8) $\mathrm{m}$ and $\mathrm{n}$ indicate nodes and $\mathrm{k}$ and $\mathrm{l}$ indicate frequencies. The function $\mathrm{G}$ is the complex conjugate of the fourier transform of the derivative of the current leaving node $m$ to the voltage at node $n$ in the time domain:

$$
G_{m, n, h}=f_{0} \int_{0}^{1 / f_{0}} \frac{\partial i_{m}}{\partial v_{n}}(t) e^{j 2 \pi n f_{0} t} d t=\mathfrak{J}\left\{\frac{\partial i_{m}}{\partial v_{n}}(t)\right\}^{*}
$$

This function needs to be calculated for $\mathrm{m}$ and $\mathrm{n}$ going from 1 to $\mathrm{N}$ and $\mathrm{h}$ going from 1 to $2 \mathrm{H}$. This means that the Jacobian with $4 \mathrm{~N}^{2} \mathrm{H}^{2}$ elements can be constructed from $2 \mathrm{~N}^{2} \mathrm{H}$ elements.

\section{B. Evaluating non-linear currents and Jacobian}

When evaluating the current through non-linear elements, an inverse fast fourier transform (IFFT) is applied to the voltages. Then the currents can be calculated in the time domain, based on the current-voltage relationships of the non-linear components, and afterwards a fast fourier transform (FFT) is used to calculate the frequency components of these currents. A similar approach is used to evaluate the fourier transform of the derivatives of the currents from equation (9).

This approach gives rise to ringing artifacts at discontinuous changes in the currents as can be seen in Fig. 4. These artifacts can easily be confused with oscillations of voltages and currents due to resonance in the circuit and is therefore highly undesirable.

The effect can be reduced by applying window functions to the frequency components of the voltage before applying the IFFT. Examples are the Hann and
Parzen window functions. The effect of these functions is also shown in Fig. 4. These functions reduce the height of the ringing artifacts but at the same time they reduce the effective number of harmonics used in the simulation. The Parzen window has a bandwidth of only one fourth of the bandwidth of a simple rectangular window but almost completely eliminates the ringing artifacts. This also means that when applying a Parzen window, four times the number of harmonics have to be used in order to get the same rise and fall times observed when using a rectangular window.

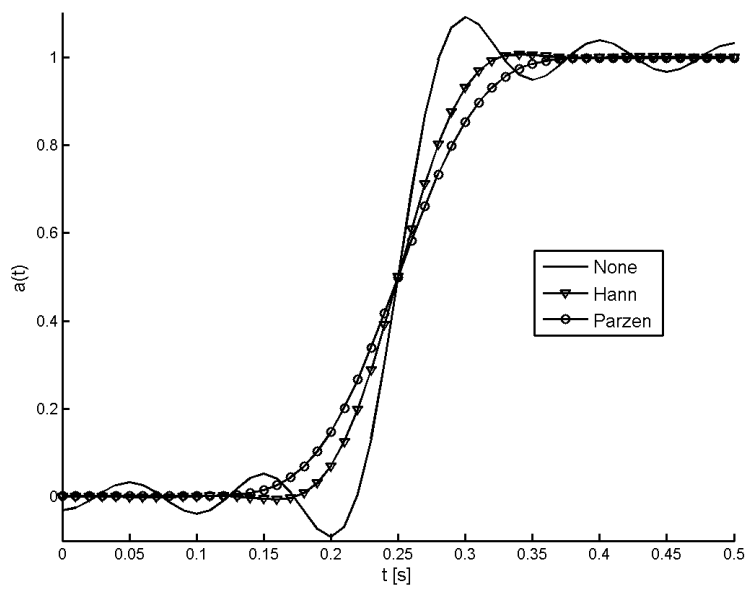

Fig. 4. Square wave approximated in the frequency domain by 10 harmonics

\section{Validation}

To validate the circuit simulator a simple single phase full-wave rectifier was simulated with both the method described in this paper and PSpice. The AC voltage source has an amplitude of $10 \mathrm{~V}$ and frequency of $20 \mathrm{kHz}$. Four D1N914 diodes were used in the rectifier. No output capacitor was used. The voltage across the load of $100 \Omega$ is shown in Fig. 5. As can be seen in the figure, the results match very well.

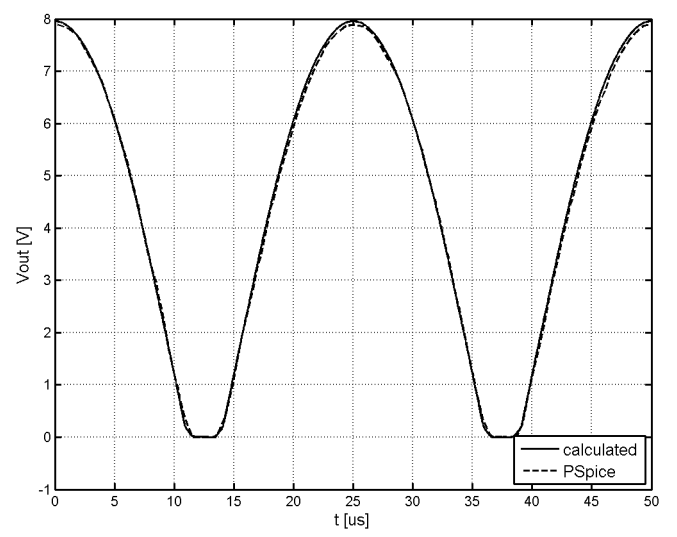

Fig. 5. Voltage across load of a simple single phase full-wave rectifier

\section{RESUlTS}

After validating the two parts of the program, it is used to determine the voltages in a buckconverter with a switching frequency of $6 \mathrm{MHz}$. The convertor is shown in Fig. 6. The conductors have a square cross-section of 
$1 \mathrm{x} 1 \mathrm{~mm}^{2}$. The DC voltage source delivers $10 \mathrm{~V}$ and the load resistance is $100 \Omega$. An inductance of $9 \mathrm{mH}$ is used. An IRF150 switch and a D1N914 diode are modelled by their current-voltage relationships. No parasitic capacitances were modelled.

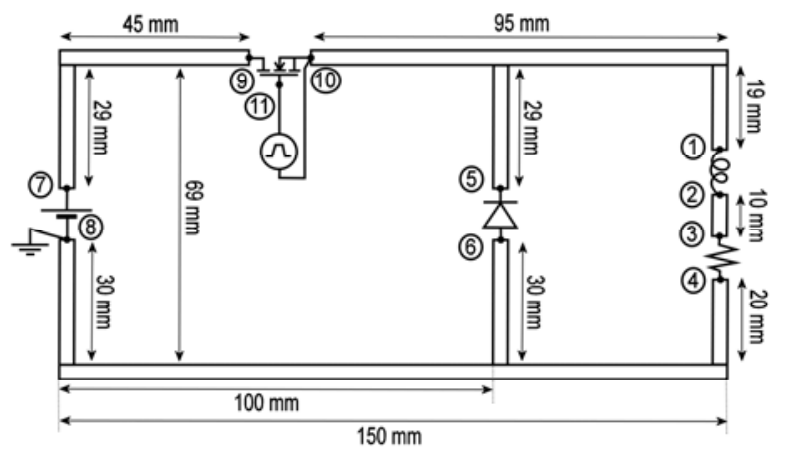

Fig. 6. Geometry of buckconvertor

A simulation was run with 120 harmonic frequencies taken into account. The window of Parzen was used. The resulting voltage in the time domain at node 1 is shown in Fig. 7.

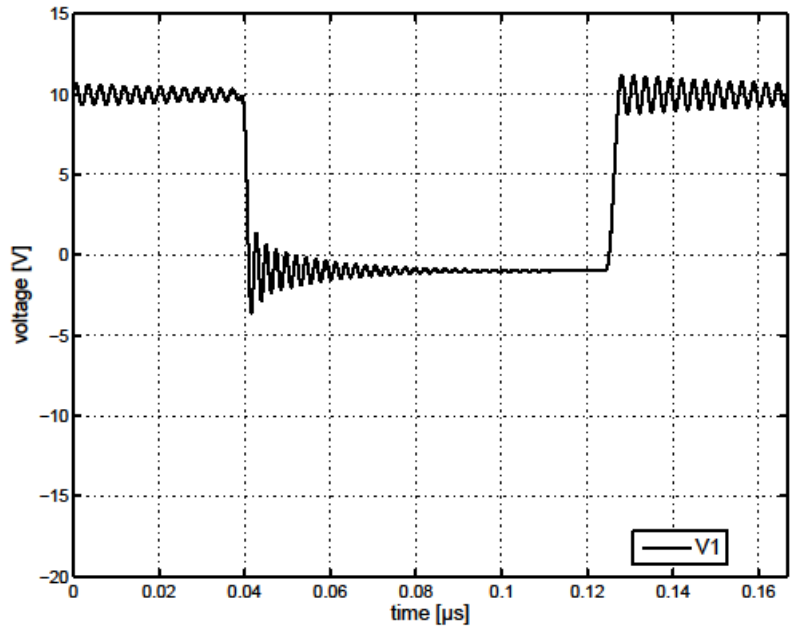

Fig. 7. Voltage at node 1 from Fig. 6 switching at $6 \mathrm{MHz}$

Clear resonance can be seen at the frequencies of 350 $\mathrm{MHz}$ and $430 \mathrm{MHz}$. These come from two standing waves visualized in Fig. 8. One occurs when the switch opens (arrow b in Fig. 8) and a second one when the switch is closed (arrow a in Fig. 8).

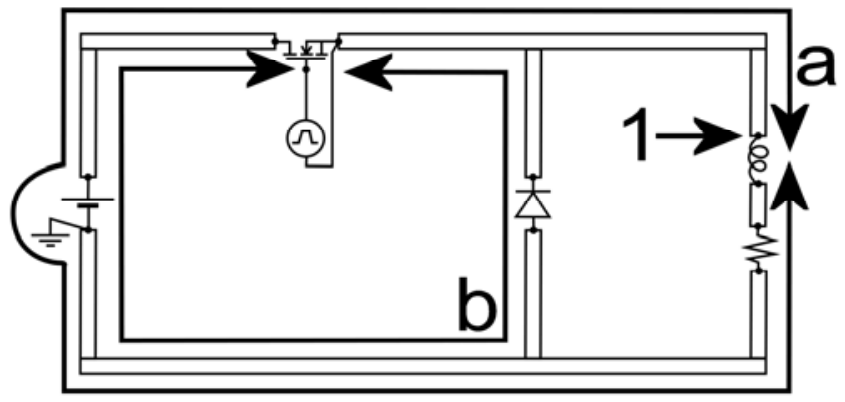

Fig. 8. Buckconvertor with visualized standing waves

\section{CONCLUSION AND FUTURE WORK}

With the method presented in this paper it is possible to accurately calculate the voltages and currents in a circuit with non-linear, non-ideal elements. The geometry of the conductors are taken into account using the full equations of Maxwell.

There are nonetheless still improvements to be made. The program now calculates its own equivalent admittance matrix of the conductors. However it can also be calculated by combining programs FastCap and FastHenry [1]. Both make use of more efficient techniques for calculating the Green functions.

Another issue is the memory use of the circuit simulator which is $\mathrm{O}\left(\mathrm{N}^{2} \mathrm{H}^{2}\right)$ with $\mathrm{N}$ the number of nodes and $\mathrm{H}$ the number of frequencies. This because of the full Jacobian being stored. As explained in section III.A, the Jacobian only consists of $\mathrm{N}^{2} \mathrm{H}$ unique elements. This can be exploited by using an iterative method rather than a direct method to calculate each step [9]. An iterative method requires only the product of the Jacobian with a vector. In that way the full Jacobian will not have to be stored and the memory requirements will only be $\mathrm{O}\left(\mathrm{N}^{2} \mathrm{H}\right)$. Reducing memory requirements is crucial as this is the biggest drawback of solving in the frequency domain.

Furthermore, by making good use of the unique structure of the Jacobian [8][9], the computation time for each step can go down from $\mathrm{O}\left(\mathrm{N}^{3} \mathrm{H}^{3}\right)$ to $\mathrm{O}\left(\mathrm{N}^{3} \mathrm{H}^{2} \log (\mathrm{H})\right)$.

\section{REFERENCES}

[1] J. Ekman, "Electromagnetic modeling using the partial element equivalent circuit method", Ph.D. dissertation, Dept. of Computer Science and Electrical Engineering, Lulea University of Technology, Lulea, Sweden, 2003

[2] W.C. Gibson, The method of moments in electromagnetics, New York, Chapman \& Hall CRC, 2008

[3] R.F. Harrington, Field Computation by Moment Methods, New York, IEEE Press, 1993

[4] J. Carlsson, (1998), A method of moments program for radiated emission and susceptibility analysis of printed circuit boards [Online], Available: http://www.sp.se/en/index/research/emc/Sidor/PCB-Mom.aspx

[5] K. Nabors and J. White, "FastCap: a multipole accelerated 3-D capacitance extraction program," in IEEE Trans. on Computer-Aided Design of Integrated Circuits and Systems, vol.10, no.11, pp.14471459, Nov 1991

[6] M. Kamon and M. Tsuk and J. White, "FastHenry: a multipoleaccelerated 3-D inductance extraction program," in IEEE Trans. On Microwave Theory and Techniques, vol.42, no.9, pp 1750-1758, Sept. 1994

[7] R.S. Elliott, Antenna Theory and Design, New Jersey, John Wiley and Sons, 2003, pp 318

[8] K.S. Kundert and A. Sangiovanni-Vincentelli, "Simulation of nonlinear circuits in the frequency domain" in IEEE Trans. Computer-Aided Design Integr. Circuits Syst., vol.5, no.4, pp. 521535, October 1986

[9] L. Sorber and M. Van Barel and L. De Lathauwer, "Unconstrained optimization of real functions in complex variables," Dept. Computer Science, K.U.Leuven, Leuven, TW Report 592, April 2011, pp 15-18 